\title{
Banalización y singularización de la muerte en la literatura concentracionaria
}

\author{
Trivialisation and singularisation of death \\ in the concentrationary literature
}

\section{Laura Miñano Mañero}

Universitat de València. Laura.minano@uv.es

Recibido: 23.06.2019. Aceptado: 11.07.2019

Resumen: En los campos de concentración nacionalsocialistas se transforma la concepción de la muerte hasta límites desconocidos en la historia. La noción arraigada en la civilización que interpreta la muerte como el principio individualizador final y determinante de la experiencia humana desaparece de manera categórica y, más bien, nos enfrentamos a una producción mecánica de cadáveres en masa. A partir del corpus testimonial legado por los supervivientes, es posible reflexionar sobre su concepción relativa a esa muerte propia y ajena. Explorando la literatura concentracionaria, además, hallamos pruebas de cómo los reclusos logran, entre esa sistemática banalización de la muerte, encontrar significado y singularizar algunos fallecimientos para, de ese modo, conseguir reafirmarse como seres humanos. Para acotar el objeto de estudio de este trabajo y asegurar un análisis contextualizado, nos centraremos principalmente en experiencias y recuerdos de supervivientes judíos del complejo Auschwitz-Birkenau.

Palabras clave: campos de concentración; Shoah; literatura concentracionaria; deshumanización; muerte.

\footnotetext{
Abstract: In Nazi concentration camps the notion of death is disfigured and exceeds every limit traditionally rooted in human civilization. In fact, the idea that interprets death as the final and definitive individualizing principle of human experience categorically disappears and, rather, we face a mechanical, massive production of corpses. Through the testimonial corpus left by survivors, it is possible to reflect on their conception of their own -and others'- death. Exploring concentrationary literature, I would suggest that prisoners succeed in overcoming this systematic trivialization of death through the search of meaning regarding other deportees' deaths. Survivors single out, personalize and claim certain fatalities as a mechanism to subvert Nazi power and assert the victims' human identity. In order to delimit the object of study and to ensure a

》 Miñano Mañero, Laura. 2019. "Banalización y singularización de la muerte en la literatura concentracionaria". Quaderns de Filologia: Estudis Literaris XXIV: 217-236. doi: 10.7203/qdfed.24.16341
} 
contextualized study, this paper mainly focuses on experiences and memories of Jewish survivors in the Auschwitz-Birkenau complex.

Keywords: Concentration camps; Shoah; concentrationary literature; dehumanization; death. 


\section{Introducción}

Los poderes totalitarios degeneran las ideas aceptadas sobre la individualidad del hombre y, al considerar a los ciudadanos como simples instrumentos para la consecución de una política nacional, logran también pervertir la relación del ser humano con su muerte. Entre ellos, el gobierno nacionalsocialista sobresale de manera decisiva por ser el único régimen que ha establecido como objetivo central de su política el fomento del desarrollo técnico y científico para poner en práctica un sistema de exterminio a nivel industrial, llevado a cabo en el campo de concentración -Konzentrationslager-. Este procedimiento de aniquilación mecánico, frío y calculado, sin embargo, no se satisface con el asesinato masivo: previamente, se busca la deshumanización y depauperación extrema de las víctimas; después, se aprovecharán de alguna manera los restos humanos para el beneficio del Reich.

Pese a que no todos los lager alemanes revelaban la misma tipología, es incuestionable que en todos ellos los prisioneros compartían una misma experiencia: la presencia constante de la muerte violenta, masiva y despersonalizada. En este trabajo, nos disponemos a analizar cómo se transforma la relación entre el hombre y la muerte en el universo concentracionario. Nuestro corpus de análisis principal se limita al legado literario de Berler, Fénelon, Klüger, Lasker-Wallfisch, Lengyel, Levi, Lustig, Nomberg-Przytyk, Steinberg y Venezia, todos ellos supervivientes judíos del complejo Auschwitz-Birkenau. Además, incorporamos también los manuscritos que el prisionero Zalmen Gradowski enterró en el campo, antes de morir. Los campos de exterminio se concibieron expresamente para aniquilar a la raza hebrea (Kogon, 2005: 283); por tanto, es plausible considerar que la relación de este colectivo con la muerte resulte la más compleja, multidimensional y paradigmática del universo concentracionario. En este trabajo defendemos la hipótesis de que los prisioneros, conscientes del objetivo deshumanizador del sistema de aniquilación nazi, intentan encontrar una razón diferenciadora que dote de significado la muerte propia y ajena. Reivindicar y singularizar el asesinato de los deportados es, a nuestro juicio, una manera de subvertir el sistema que permite a los reclusos reafirmar la identidad humana negada por el verdugo.

Nos proponemos, en primer lugar, explorar los límites que se establecen en el campo en torno a nociones tales como la espontaneidad y 
personalidad de los sujetos: examinaremos brevemente las condiciones de despersonalización a las que son sometidos los reclusos, dado que estas significan el primer paso hacia la novedosa concepción de la muerte que impregna el universo del lager. En segundo lugar, señalaremos algunas características de la literatura concentracionaria relacionadas con su valor testimonial y las necesidades concretas que llevan a los supervivientes a legar su experiencia por escrito. Trataremos de contextualizar a los autores que, en calidad de escritores-testigo, deciden destacar ciertos acontecimientos concretos de sus años de deportación por encima de otros, confiriéndoles un estatuto de autoridad especial y expresando su férreo deseo de que pasen a formar parte de la memoria colectiva.

En tercer lugar, profundizaremos en la banalización de la muerte que se lleva a cabo en el lager. Además de explicar los métodos nazis puestos en práctica para alcanzar este fin, indagaremos asimismo en el impacto personal que este proceso entraña para los autores. Más adelante, incidiremos en las estrategias de singularización que los reclusos pueden emplear para dotar de significado la muerte que les rodea -y la suya propia. En concreto, analizaremos dos sucesos - cada uno de ellos relacionado con los últimos momentos de una prisionera- que han sido narrados por un gran número de supervivientes y colectivamente señalados como ejemplos singulares de temple y entereza. Finalmente, expondremos las conclusiones de este análisis, que versarán sobre la capacidad de resistencia de las personas durante su último aliento, y la reivindicación realizada por aquellos otros que les prestan la voz para destacar, diferenciar, y dotar de significado sus muertes.

\section{Deshumanización}

Los campos de concentración son los laboratorios donde el régimen nacionalsocialista aspira a la dominación total de todos aquellos seres humanos que no encajan en su ideal racial o desafían de alguna forma el concepto del sistema. En el lager se busca destruir la pluralidad y la diferenciación de los sujetos, organizando la humanidad de manera conjunta, como si se tratara de una identidad única y nunca cambiante de reacciones controladas, de manera que, al final, todos los ciudadanos resultan superfluos e intercambiables. Para obtener estos sujetos-masa, según Arendt, es necesario aniquilar cualquier forma de espontaneidad, 
dado que esta constituye la expresión más natural del comportamiento humano. De ese modo, se debe recluir a las víctimas en un espacio totalmente controlado y ajeno a la realidad exterior. La dominación absoluta, de hecho, requiere "el aislamiento respecto del mundo de todos los demás, del mundo de los vivos en general" (Arendt, 2006: 590). El lager nacionalsocialista, afirma la autora, es el único espacio en el que es posible desintegrar completamente la identidad humana y llegar a la sumisión incondicional de las personas (2006: 589-592).

El proceso de deshumanización comienza inmediatamente tras la llegada al campo, con la ceremonia de bienvenida -"Empfangszeremonie" (Kogon, 1974: 73)-, que consiste en arrebatar al deportado todo vínculo con su vida anterior. Además de violar su dignidad física y despojarles de cualquier bien material que todavía hubieran conservado, se procede a la transformación de su aspecto exterior: se rapan sus cabezas y se sustituyen sus vestimentas por andrajos. En última instancia, el prisionero pierde su nombre propio para pasar a figurar como un simple número. Inmediatamente, la despersonalización surte efecto, y es difícil reconocer a amigos o familiares. En palabras de la deportada polaca Nomberg-Przytyk: "I was looking for acquaintances among those transformed figures, and truly, I did not recognize anybody ${ }^{1 "}$ (1985: 14).

Definitivamente, tal como percibe Paul Steinberg al llegar: "todos los seres humanos que me rodean son intercambiables" (2004: 111). Así, desde el primer momento, los autores vislumbran la voluntad del verdugo de aniquilar la pluralidad y personalidad individual. Primo Levi reflexiona, igualmente, sobre las implicaciones del infame tatuaje que sustituía sus nombres en Auschwitz: "Es la marca que se imprime a los esclavos y a las bestias destinadas al matadero, y es en lo que os habéis convertido" (1989: 112).

La violencia y la animalización son elementos constantes e inherentes al lenguaje de los perpetradores desde que los prisioneros llegan al campo. Las agresiones físicas conllevan también una lengua excesivamente degenerada y vulgar. La llegada de los reclusos incorpora un discurso de bienvenida a cargo de algún SS. Steinberg recuerda su tono amenazador y la repetición exhaustiva de la expresión "Von hier geht es

\footnotetext{
${ }^{1}$ Algunas obras se citan en inglés por no haber sido traducidas al español. En este caso, el texto original es polaco.
} 
nur durch den Schornstein raus" - de aquí solo se sale por la chimenea(2004: 81).

Los intentos de deshumanizar al prisionero impregnaban la lengua de poder: Los deportados eran Arbeitstücke (piezas de trabajo) (Millu, 2005: 26), y los recién llegados se conocían, peyorativamente, como Zugang (transporte) (Levi, 1989: 36). La animalización y las comparaciones con bestias también eran frecuentes en el lenguaje del perpetrador $-\mathrm{y}$, en ocasiones, asimiladas por los deportados-. Por ejemplo, Levi recuerda que en el campo se utilizaba el término alemán fressen, que sirve para describir el comer de los animales, mientras que, en el mundo exterior, essen es el vocablo que se aplica a los hombres (1989, 81). Venezia evoca la imagen del S.S. "aullando como una bestia furiosa: 'Arbeit!', 'A trabajar, cerdos -Schweine- judíos' " (2010, 78). Parece que, comenzando con este detallado proceso deshumanizador, el verdugo llegará también a corromper la relación del ser humano con la muerte, desafiando todas las implicaciones atribuidas tradicionalmente a esta noción.

\section{El escritor-testigo}

La deshumanización, la presencia de la muerte y el lamento por los caídos son temas constantes en el corpus testimonial legado por los supervivientes. La información oficial de los campos que se ha conservado queda velada por el sesgo y el ostracismo, así como por un lenguaje excesivamente eufemístico y ajeno a la realidad. Para poder aproximarnos a la experiencia concentracionaria, por lo tanto, explorar los escritos de los deportados es imprescindible. Sánchez Zapatero (2010: 41) afirma que la escritura sirve para transmitir y materializar el recuerdo; incide, además, en la idea de que la memoria colectiva se sustenta parcialmente en las interpretaciones personales de la historia, por lo que parece acertado que "en determinadas ocasiones, ser víctima, testigo y superviviente sea suficiente legitimación para alzar la voz y contar lo vivido" (43). En el caso de la literatura concentracionaria, además, los textos del escritor-testigo constituyen prácticamente la única fuente de información relativa a un acontecimiento significante y ensombrecido, de manera que se convierten en la única forma de interpretación, reacción y superación histórica. Sánchez Zapatero destaca el imperativo moral que, a raíz de esta situación, invade al superviviente: "de ahí que 
reflexionar sobre lo vivido [...] se convierta en una obligación para con la sociedad, en un compromiso con la verdad que se intenta ocultar" (41).

La compulsión de las víctimas por narrar la experiencia colectiva nace desde el momento en que comienzan a sentir el peso del verdugo. Borwicz (1996) dedica uno de los primeros estudios a la literatura de los judíos polacos nacida bajo la ocupación nazi, analizando documentos como los escritos en muros de prisión, los poemas compuestos en el lager, o las últimas cartas de los condenados a muerte. Tanto en los textos de quienes perecieron como en los de los supervivientes es posible apreciar su pretensión de memoria ejemplar que, según Todorov (2010: 275), se caracteriza por el intento de explicar el pasado con vistas a construir un futuro en el que no se vuelvan a cometer los mismos errores. Así lo ha explicitado Olga Lengyel:

In setting down this personal record I have tried to carry out the mandate given to me by many fellow internees at Auschwitz who perished so horribly. This is my memorial to them. [...] I want the world to read and to resolve that this must never, never be permitted to happen again (1995: 224-225).

En esta cita, la superviviente enlaza su voluntad de memoria ejemplar con otra de las preocupaciones principales de los autores: la de rendir homenaje y dar voz a todos los compañeros caídos. Con frecuencia, los autores son conscientes de la laguna que existe irremediablemente en el corpus testimonial. Siguiendo a Berler, "es verdad que sobreviví, [...] pero: ¿qué prueba esto? Quienes podrían haber acusado más duramente al totalitarismo nazi no volvieron" (2001: 67).

Primo Levi también siente estar hablando por delegación, para manifestar la experiencia de los que no han vuelto. Insiste en una idea: "lo repito... no somos nosotros, los supervivientes, los verdaderos testigos...". Según el italiano, "los testigos integrales, aquellos cuya declaración habría podido tener un significado general" son los hundidos, "quien ha visto a la Gorgona" (1989: 72). La experiencia concentracionaria de aquel que ha llegado a la liberación de los campos con vida es, por lo tanto, incompleta. La imposibilidad de narrar la muerte humana les impide referir enteramente el destino compartido por millones de víctimas. 
No es de extrañar que, dadas las circunstancias, la muerte se convierta en una de las cuestiones centrales en la literatura del lager. Insistiendo en la búsqueda de esa memoria ejemplar, los supervivientes exigen que el sufrimiento de los caídos no haya sido en vano. Igualmente, el imperativo moral que les guía obliga a los autores a reflexionar sobre las condiciones en las que se produce la muerte en los campos.

\section{Banalización de la muerte}

En Auschwitz,

existe la falta de esperanza apática, encarnada en el fenómeno de los "musulmanes", las personas que, en el campo de concentración, habían perdido el instinto de conservación y reaccionaban como autómatas, casi como autistas. Se los tenía por casos perdidos, ningún musulmán podía sobrevivir largo tiempo (Klüger, 1997: 109).

Los Muselmänner, los “no-hombres” (Levi, 1999: 96), son el máximo exponente del proceso de deshumanización que sufre la víctima en el campo. La expresión, de origen incierto, sirve para describir a los prisioneros absolutamente extenuados que, aunque todavía respiran, no pueden considerarse como vivos. Según algunos testimonios, de hecho, los funcionarios del campo trataban a estos prisioneros como si ya estuvieran muertos. Siguiendo a Nomberg-Przytyk, por ejemplo, las deportadas condenadas en una selección eran conducidas hasta la antesala de la muerte sin ropa, para no tener que cargar con sus vestimentas después de haberlas asesinado (1985: 56). En la misma línea, Oliver Lustig cuenta que el verdugo nazi marcaba a algunos presos recién llegados con una L en la espalda; las víctimas pensaban que significaba 'Lager', pero en realidad se trataba de la inicial de Leiche, 'cadáver', que aseguraba la exterminación inmediata de su portador (1982: 190-191). Así, parece que el poder absoluto consigue, en primer lugar, difuminar la frontera entre la vida y la muerte. La superviviente Hanna Lévy-Hass ha incidido en esta idea:

No estamos muertos, pero somos unos muertos. Han matado en nosotros no sólo el derecho a la vida presente, [...] sino que también han conseguido con sus métodos sádicos y perversos matar cualquier sensación de una vida humana anterior, cualquier sentimiento propio de unos 
seres normales dotados de un pasado normal, incluso la conciencia misma de haber tenido en algún momento una existencia humana digna de ese nombre (2006: 85).

El proceso de deshumanización, de ese modo, se consolida como el primer paso hacia la banalización de la muerte. El perpetrador trata a las víctimas como bestias de carga, con un "tono de voz cargado de odio que suprime lo humano del así tratado o así gritado y lo define o la define como un objeto" (Klüger, 1997: 115). Al negar la pertenencia de los presos a la especie humana, el verdugo se libera de cualquier responsabilidad moral por sus atrocidades:

Lo primero que aprendí en el campo, y nos lo repitieron constantemente, fue que no éramos más que cuerpos. [...] Ésa fue la primera obscenidad y de ella se derivaron todas las demás, entre otras la pérdida de la solemnidad trascendente de la muerte (Berler, 2001: 63).

En el campo de concentración se disipa la frontera demarcatoria natural que separa a los vivos de los difuntos, pues la existencia del musulmán se caracteriza por un nivel de depauperación, aislamiento y abandono que difícilmente puede asociarse a la vida. En efecto, Levi defiende que estos individuos son, más bien, "fantasmas que caminan" (2015: 104105); Piera Sonnino se refiere a ellos con frecuencia como "esqueletos" (2018: 89); y para Borowski son los "muertos vivientes" (2004: 85). Por ello, parece difícil considerar que, cuando estas personas finalmente perecen, su defunción signifique la negación de la vida, pues desde mucho antes las víctimas ya transitaban en una zona incierta y difusa. En definitiva, la muerte ya no es el acontecimiento que, según Sofsky, separa los dos estados fundamentales y antagónicos de la experiencia humana (2016: 303). Podemos preguntarnos, por lo tanto, si la muerte que se sufre en el campo de concentración es realmente una muerte. En este sentido, a propósito de los musulmanes, Levi indica que "se duda en llamarlos vivos: se duda en llamar muerte a su muerte" (1999: 96). También Lévy-Hass se plantea una cuestión similar:

¿Cómo permitir que tu cuerpo y tu alma se pudran y se confundan con su propia inmundicia, desaparecer lenta pero irrevocablemente por inanición total, hundirse en la nada, devorado por el pus, la pestilencia y pasando por todas las fases de la descomposición antes de reventar? 
Porque es exactamente eso: aquí uno no se muere, revienta literalmente (2006: 129).

Además, la sistematización de un proceso de asesinato industrial, masivo, en cadena, lleva a considerar los centros de exterminio, más bien, como "fábricas de cadáveres" (Arendt, 2006: 616) en las que se desposee al hombre de cualquier noción tradicional que conciba la muerte como el principio individualizador y determinante de la experiencia humana: "el exterminio de las personas individuales ha sido absorbido por el exterminio general", a través de una "muerte regular, sistemática" (Gradowski, 2017: 70). Schlomo Venezia, que fue obligado a trabajar en los crematorios de Auschwitz, meditó sobre las víctimas de las cámaras de gas: "su muerte lo era todo salvo una muerte dulce. Era una muerte inmunda, sucia. Una muerte forzada, difícil y distinta para todos" (2010: 83). Zalmen Gradowski, empleado en la misma brigada que Venezia, brinda una reflexión análoga:

Esos cuerpos rebosantes de vida, sorprendentes, se extenderán por la tierra como criaturas asquerosas, rodando por la suciedad y la inmundicia; esos cuerpos puros, alabastrinos, se ensuciarán de la mierda humana (2017: 147).

En definitiva, el sistema nazi busca desvirtuar cualquier concepción trascendental de la muerte. De hecho, Primo Levi considera que el procedimiento seleccionado para la exterminación en masa era ostensiblemente simbólico: "había que usar, y se usó, el mismo gas venenoso que se usaba para desinfectar las estibas de los barcos y los locales infestados de chinches" (1999: 206). El poder totalitario pretende, además, borrar por completo la huella de las víctimas - "no quedará ninguna señal, nada de todas esas personas que están aquí, [...] porque dentro de poco van a ser arrancadas de cuajo de sus raíces como si nunca hubieran nacido" (Gradowski, 2017: 148)-, pues los cadáveres son ultrajados y empleados como materias primas para el aprovechamiento del Reich. Oliver Lustig recuerda que la única certeza absoluta del prisionero era la muerte, y opone la homogeneidad, monotonía y regulación extrema que caracterizaban la existencia del deportado a la multiplicidad de rostros que podía adoptar la muerte -"numai der Tod, moartea, era inimaginabil de variată", 'tan solo der Tod, la muerte, era inimaginablemente variada'-(1982: 283). En efecto, aunque las cámaras de gas simbolicen 
la cúspide del proceso de banalización, es necesario considerar que el asesinato era una constante absoluta, pero también variable y caprichosa. Su presencia arbitraria y omnipresente, es decir, el hecho de que todo recluso supiera que en cualquier momento y por cualquier motivo, por absurdo que fuera, alguien podía arrebatarle la vida -o lo que quedara de ella- reforzaba la idea de que la muerte era absolutamente insignificante. Incluso a aquellos que consiguieron sobrevivir al campo, este trauma les persiguió cuando regresaron al mundo de los vivos:

Nunca más he podido recuperar el comportamiento respetuoso y compasivo ni la presencia de ánimo que hay que mostrar por los muertos. Tengo que hacer un esfuerzo para obligarme a expresar mi aflicción a sus familiares. Mi propia muerte me es familiar, conozco su rostro. La he vislumbrado con frecuencia. La angustia metafísica me es tan ajena como una galaxia lejana. Todo esto me excluye de la comunidad (Steinberg, 2004: 126-127).

\section{Singularización de la muerte}

La literatura concentracionaria nos demuestra, sin embargo, que el objetivo del sistema nazi de banalizar la muerte de las víctimas no surte efecto en todos los casos. Los oprimidos, más bien, conceden una significancia especial a algunas ejecuciones del campo que, glorificadas, pasan a convertirse en pilares esenciales del canon testimonial. Los autores reivindican y reclaman la caída de sus compañeros que, con el último aliento, desafían al régimen totalitario. Estas experiencias subversivas, así como el procedimiento de perpetuarlas a través de la escritura para las generaciones futuras, simbolizan el esfuerzo de los deportados por reafirmar la identidad humana de las víctimas. Durante el período de funcionamiento de Auschwitz-Birkenau, el relato de dos tragedias concretas, que individualizan el final de dos mujeres, ha sido reiterado por los supervivientes con insistencia. Los años transcurridos entre el acontecimiento y la redacción de la obra, los mecanismos específicos de la memoria y el impacto del trauma en los autores llevan a la inevitable divergencia en los detalles del suceso. Ahora bien, a través de un análisis de las múltiples versiones, es posible reconocer la obstinación de los supervivientes por distinguir esas muertes.

La primera de las historias se relaciona con las ejecuciones en los campos de concentración. Ante la fuga de un prisionero, los nazis 
desplegaban todos sus recursos para encontrarle y devolverle con vida al campo. Que una evasión resultara exitosa era decididamente difícil, pues no solo había que contar con ayuda interna en el campo que facilitara la oportunidad de salir. Una vez fuera, era imprescindible disponer de contactos civiles que, no envenenados por el fanatismo del sistema, protegieran a las víctimas arriesgando su propia integridad física. En la mayoría de los casos, los prisioneros eran delatados y devueltos al lager. La S. S., entonces, organizaba una ejecución ejemplar para los evadidos, que todos los prisioneros debían presenciar obligatoriamente. Los nazis no comprendían que, tratando de disuadir a los reclusos de la tentativa de fuga y de demostrar humillantemente su poder absoluto sobre los deportados, en realidad estaban, ellos mismos, singularizando la muerte de estas víctimas.

La historia de Mala Zimetbaum ha sido narrada por muchos supervivientes. Los relatos concuerdan en que Mala fue una joven resistente judía belga, de ascendencia polaca. Gracias a su perfil políglota consiguió que le asignaran la función de intérprete en el campo, de modo que la joven se convirtió en el enlace que permitía la comunicación directa entre las víctimas y el verdugo. La artista francesa Fania Fénelon, una de las prisioneras privilegiadas de la orquesta del campo, pudo estrechar el contacto con ella:

[Mala] es mucho más que un nombre, es ya una leyenda. [...] Se ignora el motivo de que los S. S. le hayan otorgado su confianza sin que tenga que dar a cambio las contrapartidas que tanto aprecian: delaciones y denuncias, celo durante las selecciones, etc. Tal vez porque es una joven valerosa, silenciosa, tranquila y eficaz. En realidad, sin que ellos se den cuenta, impone su voluntad y los domina. Las internadas la estiman y la quieren. Todas tenemos una confianza ciega en ella. [...] Las deportadas acuden a ella cuando se encuentran en dificultades o tienen algún problema (1981: 215-217).

Sara Nomberg-Przytyk también recuerda su entrega y valentía:

She was willing to undertake even the riskiest tasks, and she always brought them off. [...] With her skill and her daring, she had managed to save the lives of many women (1985: 101). 
Mala pudo emplear su influencia en el campo para organizar una tentativa de evasión junto a otro prisionero, Edek Galinski ${ }^{2}$. Consiguieron escapar del campo, pero unos días después fueron capturados y devueltos al lager. Cuando iban a ahorcarla públicamente frente a todas las reclusas, la joven belga decidió adueñarse de su última expiración:

Mala había decido vivir su propia muerte. [...] Había logrado hacerse con una hoja de afeitar. Al pie de la horca, se cortó la arteria de una muñeca. El SS que hacía de verdugo trató de quitarle la cuchilla, y Mala, ante todas las mujeres del campo, le golpeó la cara con la mano ensangrentada. Inmediatamente acudieron otros militares, enfurecidos: ¡una prisionera, una judía, una mujer, se había atrevido a desafiarlos! (Levi, 1989: 145-146).

Las versiones coinciden en que la condenada trató de suicidarse antes de ser ejecutada y abofeteó a su verdugo. Olga Lengyel comenta también que, cuando le obligaron a ponerse un cartel que indicaba su delito ${ }^{3}$, Mala se negó, desafiando públicamente al sistema establecido (1995: 136). Al cortarse las venas, la joven afirmó la última libertad y manifestó su capacidad de espontaneidad y resistencia; en definitiva, demostró que la humanidad que le negaba el totalitarismo todavía estaba en sus manos. La S. S. no podía permitirlo. Fania Fénelon, que se encontraba muy cerca, detalla los últimos instantes de Mala de la siguiente manera:

Los S. S. se precipitan, la derriban, la pisotean, le ponen un torniquete. La quieren viva. Le atan los brazos detrás de la espalda, se la llevan. Mala se cae, vuelve a levantarse y nos grita:

-¡Rebelaos! ¡Rebelaos! Sois miles. ¡Atacadlos! Son unos cobardes. E incluso si os matan, será mejor para vosotros. ¡Moriréis libres! ¡Rebelaos! (1981: 223) .

\footnotetext{
${ }^{2}$ La obra de Wieslaw Kielar, Anus mundi: 1500 days in Auschwitz-Birkenau (1980), amigo cercano de Edek, es la que retrata con mayor detalle la fuga de los prisioneros. ${ }^{3}$ En una ejecución por tentativa de fuga, era frecuente colgar a los prisioneros una cartulina en el cuello con sentencias humillantes como " 'Hurrah! Ich bin wieder da!', es decir, ‘¡Hurra! ¡Estoy aquí otra vez!’” (Kertész, 2006: 163).

${ }^{4}$ Anita Lasker-Wallfisch, violoncelista de la orquesta, respalda la versión de Fénelon y añade que Mala lanzó insultos a la S.S. durante los últimos momentos (2000: 82).
} 
Lengyel refiere unas últimas palabras diferentes: "Still the girl, half dead, with a pierced eye and a gory face, raised herself and cried out: 'Courage, friends' 'They will pay! Liberation is near!' ' (1995: 137). Según el testimonio de Hanna Kampel, recogido por Borwicz, Mala gritó a las prisioneras: "les jours que je viens de passer en liberté m'ont donné une certitude absolue que l'Allemagne se trouve au seuil même de sa chute et que la libération est toute proche" (1996: 245).

El final de Mala puede diferir en pequeños detalles según cada relato; lo esencial, sin embargo, permanece de forma constante en todas las versiones. La joven judía decidió no ser una víctima pasiva y mostró su autonomía durante el último aliento, animando a las demás deportadas. Su muerte ha sido narrada por muchas personas: se menciona también, por ejemplo, en el testimonio de la resistente polaca Seweryna Szmaglewska (2006: 367), uno de los primeros en ser publicados al finalizar el conflicto bélico. La leyenda de Mala llegó incluso a otros campos con la evacuación de los prisioneros de Auschwitz hacia el corazón del Reich; así, su historia resuena igualmente en el testimonio de Lise London, deportada a Ravensbrück (1997: 490). Los supervivientes han deseado singularizar su muerte e incorporarla al legado de la Shoah como un momento de especial relevancia en la historia de los campos de concentración.

Otras demostraciones de resistencia atentan contra la idea generalmente aceptada de que las víctimas iban al crematorio dócilmente. Un ejemplo individual de subversión frente a la muerte que ha impregnado el legado literario del Holocausto concierne a una bailarina. El historiador y antiguo deportado de Buchenwald, Eugen Kogon, mencionó en su obra de referencia, Der SS-Staat (1974), a una artista italiana a quien, en el camino a la cámara de gas de Auschwitz, el S. S. Schillinger obligó a bailar desnuda. En el momento preciso, la bailarina arrebató al S. S. el revólver y le disparó, hiriéndole fatalmente. A continuación, le dispararon a ella también, de manera que, al menos, escapó al gas (167). Hermann Langbein refiere las palabras de Höss, el comandante de Auschwitz, respecto al suceso. Según él, en octubre de 1943 un Transport de Bergen-Belsen llegó a Auschwitz para ser gaseado. En la antecámara donde debían desnudarse los condenados, surgió una revuelta. Antes de que llegaran los refuerzos, Schillinger fue disparado por una mujer a quien intentaba desnudar. Höss afirmó que dio la orden de disparar, uno a uno, a los rebeldes (2004: 118-119). 
Sara Nomberg-Przytyk dedica un capítulo, Revenge of the dancer, a narrar el acontecimiento de la bailarina. Cuenta que la historia se la relató una joven prisionera que escapó a las cámaras de gas y apareció en la enfermería, donde ella trabajaba. Según esta versión, se trataba de una bailarina francesa, "an unusually beautiful woman, very pleasant and courteous" (1985: 108). Cuando los alemanes les ordenaron que se desvistieran, la joven se negó:

She was the only one who did not get undressed. As SS man, apparently the commandant of the guards, approached her. "Beautiful girl, take off your suit," he said quietly coming close and closer to her. Then, all of a sudden, with a rapid movement, she grabbed the pistol out of his holster and shot straight at him. After that, she took three steps backward and shot at the SS men who were running all over the place. She saved the last bullet for herself. She fell to the ground (198-109).

El prisionero polaco Tadeusz Borowski, que trabajó en las cámaras de gas, se encuentra en una posición más legítima para narrar la historia. En su obra, afirma que fue otro prisionero del Sonderkommando ${ }^{5}$ quien le relató lo sucedido. Debido a las funciones que desempeñó en el campo, es posible que, en realidad, utilice una estrategia de distanciamiento narrativo para referir lo que vio él mismo. En esta versión, se trataba de un cargamento de judíos polacos que, tras el levantamiento, acabaron en las cámaras de gas:

Our Polish Jews knew what was up. And so the whole place swarmed with S.S., and Schillinger, seeing what was going on, drew his revolver. But everything would have gone smoothly except that Schillinger had taken a fancy to a certain body. [...] So he walked up to the woman and took her by the hand. But the naked woman bent down suddenly, scooped up a handful of gravel and threw it in his face, and when Schillinger cried out in pain and dropped his revolver, the woman snatched it up and fired several shots into his abdomen. The whole place went wild. The woman fired once again, this time at the chief, wounding his face (1986: 145-146).

Zalmen Gradowski (2017: 132-133), también miembro del Sonderkommando, afirma haber observado con sus propios ojos aquel "acto heroico"

\footnotetext{
${ }^{5}$ Comando especial, prisioneros judíos obligados a trabajar en las cámaras de gas.
} 
que le "hace inclinar la cabeza como señal de respeto y de honor". Según su testimonio, una bailarina de Varsovia joven y valiente le quitó un revólver al S. S. Quakernack y disparó a Schillinger. En la versión de Gradowski, el coraje de esta prisionera impulsó a la insurrección: "su acto armó de valor a otras mujeres valientes que empezaron a golpear, a lanzar botellas y otros objetos parecidos a las caras de aquellas bestias enfurecidas, exasperadas". Michel Borwicz recoge, de los archivos de la Comisión central por la historia judía en Cracovia, un testimonio diferente. Según esta fuente, la artista -una actriz-realiza, antes de morir, una reflexión en voz alta que, como en el caso de Mala, reivindica su autonomía personal frente a la muerte:

L'actrice Lola Lipman conduite, au milieu d'un groupe de condamnés, dans les chambres à gaz d'Auschwitz II (Birkenau), refusait de se déshabiller. [...] Schillinger, sadique notoire, braqua alors son revolver contre cette femme. Elle sauta sur lui, fit tomber son revolver par terre, le ramassa et tira, blessant gravement le bourreau ; il mourut une heure plus tard. Pénétrant dans la chambre à gaz, cette femme dit: J'ai pris ou moins ma petite parcelle de vengeance; maintenant mon corps brûlera tranquillement (1996: 245).

Como en la experiencia de Zimetbaum, los detalles de la muerte de esta protagonista difieren en función de la versión. Aunque según Borwicz se trata de una actriz llamada Lipman, está generalmente aceptado que en realidad fue una bailarina judía polaca, Franceska Mann, quien disparó a Schillinger (Bartrop, 2016: 177-178). En realidad, no son las condiciones concretas de la muerte, ni su propio nombre o país de procedencia lo que dotan de significado a la experiencia de la bailarina. El aspecto más relevante es que el corpus testimonial reitere con insistencia las últimas acciones de esta figura, algo indefinida, que se rebela antes de ser asesinada para afirmar a sí misma, a los que la rodean y a todos aquellos que conocerán los acontecimientos, la humanidad de las víctimas. Cada versión refiere una muerte distinta -bien se dispara a sí misma, bien la abaten a tiros o bien la reducen e introducen en la cámara de gas-, pero en todas ellas, como mínimo gritando, la bailarina se niega a entregarse dócilmente, y eso es lo que dota su expiración de un valor singular.

En otras palabras, lo relevante tanto en el caso de Mala como en el de la bailarina es que sus actos de resistencia se oponen a la muerte 
sumisa, indiferente e insignificante que impera en el campo. Los demás prisioneros glorifican sus muertes con el fin de recobrar la esperanza y el ánimo, el primer paso imprescindible para evitar convertirse en musulmanes y, en última instancia, reconquistar la esencia humana que el sistema les niega. Las palabras del superviviente Wieslaw Kielar describen la profunda influencia inmediata que ejerció el ejemplo de la bailarina:

The incident passed on from mouth to mouth and embellished in various ways grew into a legend. Without doubt this heroic dead by a weak woman, in the face of certain death, gave moral support to every prisoner. We realized all at once that if we dared raise a hand against them, that hand might kill; they were mortal, too. [...] Reaction came swiftly; prisoners straightened up, hope grew once more. A spontaneous, although still weak, campaign of self-defense was born (1980: 178-179).

\section{Conclusión}

En los campos de concentración, se lleva a cabo un proceso de deshumanización de los reclusos. Desafiando los límites tradicionalmente impuestos a la concepción de la muerte, el régimen desvirtúa toda la trascendencia y solemnidad atribuida a esta noción, que en la civilización se interpreta como el último principio individualizador de la experiencia humana. Para ello el opresor emplea todo tipo de medios, tanto técnicos como organizativos e incluso lingüísticos. Los autores del corpus testimonial reflexionan sobre si el final que espera a las víctimas de los campos de concentración puede llamarse muerte o si, más bien, se trata de algo diferente. De hecho, el fenómeno del musulmán muestra que las víctimas transitan, antes de perecer, por una zona incierta en la que convergen la vida y la muerte, dos estados que, fuera del universo concentracionario, son antagónicos por definición.

La singularidad del Holocausto implica, además, el deseo de borrar cualquier huella del paso por la historia del pueblo judío. Aunque es innegable que en el lager todos los prisioneros sufren y mueren por igual, la raza hebrea queda sometida a una política genocida de magnitud total. En consecuencia, la omnipresencia de la muerte contribuye a su banalización y se convierte en la herramienta última de subyugación del prisionero. En esta particularidad puede radicar una de las causas por las que a los reclusos judíos les sobreviene una voluntad especial de do- 
tar de significado la exterminación masiva que no pueden evitar de ninguna manera. La composición de nuestro corpus, en efecto, se articula en torno al deseo de indagar sobre esta singularidad, pues fueron dos prisioneras judías quienes decidieron reclamar su propia muerte como forma de resistencia en el último momento $\mathrm{y}$, al hacerlo, se convirtieron en ejemplos de insubordinación. De hecho, los supervivientes han considerado que sus experiencias debían inmortalizarse en el legado histórico; por ello, han decidido relatar sus muertes y las han definido como intentos de reafirmar la dignidad humana que les negaba el verdugo.

A pesar de las diferencias en los detalles de cada versión, propios del funcionamiento caótico de la memoria colectiva y la transmisión de la información, podemos observar que el sentido de las historias, esto es, su articulación como ejemplos paradigmáticos de esfuerzo por singularizar la identidad propia y la resistencia última frente a la injusticia del verdugo, se mantiene en la memoria de los testigos. Sea como fuera que se desarrollaron exactamente los acontecimientos, el hecho es que la memoria colectiva ha decidido convertir al eslabón más débil del lager, la mujer judía, en el más fuerte símbolo de subversión. Además, la persistencia de estos recuerdos empuja a los supervivientes a compartirlos para la posteridad.

Del análisis realizado podemos inferir que toda la política opresora de los campos se articula en torno a la muerte. Es decir, el objetivo de exterminar a un pueblo es el que da forma a todas las estrategias y técnicas empleadas. Sin embargo, al convertir la muerte en destino único y universal de todos los presos, se genera de modo inesperado la posibilidad de que la muerte se convierta en un principio individualizador para esos prisioneros. De ese modo, la propia política diseñada para la exterminación en masa crea singularidades imperecederas.

\section{Bibliografía}

Arendt, Hannah. [1951] 2006. Los origenes del totalitarismo. Madrid: Alianza. Bartrop, Paul. 2016. Resisting the Holocaust: Upstanders, Partisans, and survivors. California: ABC-CLIO.

Borwicz, Michel [1973] 1996. Écrits des condamnés à mort sous l'occupation nazie. París: Gallimard.

Kertész, Imre. [1975] 2006. Sin destino. Barcelona: Acantilado.

Kielar, Wieslaw. [1972] 1980. Anus mundi: 1500 days in Auschwitz Birkenau. Nueva York: Times Books. 
Langbein, Hermann. [1995] 2004. People in Auschwitz. North Carolina: University of North Carolina Press.

Lévy-Hass, Hanna. [1946] 2006. Diario de Bergen-Belsen 1944-1945. Barcelona: Galaxia Gutenberg.

London, Lise. [1995] 1997. La madeja del tiempo: memoria de la resistencia. Madrid: Ediciones del Oriente y del Mediterráneo.

Millu, Liana. [1947] 2005. El humo de Birkenau. Barcelona: Acantilado.

Sánchez Zapatero, Javier. 2010. Escribir el horror: Literatura y campos de concentración. Barcelona: Editorial Montesinos.

Sofsky, Wolfgang. [1997] 2016. La organización del terror: los campos de concentración. Buenos Aires: Prometeo Libros.

Sonnino, Piera. [1960] 2018. La noche de Auschwitz. Madrid: Ardicia.

Szmaglewska, Seweryna. [1945] 2006. Una mujer en Birkenau. Barcelona: Alba Editorial.

Todorov, Tzvetan. 2010. Le siècle des totalitarismes. París: Éditions Robert Laffont.

Toran, Rosa. 2005. Els camps de concentració nazis. Barcelona: Edicions 62.

\section{Corpus de análisis}

Berler, Willy. 2001. Superviviente del infierno: el sobrecogedor testimonio de un hombre que salió con vida de Auschwitz. Barcelona: Planeta.

Borowski, Tadeusz. [1946] 2004. Nuestro hogar es Auschwitz. Barcelona: Alba Editorial, S. L.

Fénelon, Fania. [1976] 1981. Tregua para la orquesta. Barcelona: Editorial Loguer.

Gradowski, Zalmen. 2017. Me encuentro en el corazón del infierno: Manuscritos de un Sonderkommando hallados en Auschwitz. Oświęcim: Museo Estatal de Auschwitz-Birkenau.

Klüger, Ruth. [1992] 1997. Seguir viviendo. Barcelona: Galaxia Gutenberg. Lasker-Wallfisch, Anita. [1996] 2000. Inherit the truth: a memoir of survival and the Holocaust. Nueva York: Thomas Dunne Books.

Lengyel Olga. [1945] 1995. Five chimneys: A woman survivor's true story of Auschwitz. Chicago: Academy Chicago Publishers.

Levi, Primo. [1961] 2015. Así fue Auschwitz. Barcelona: Ediciones Península. Levi, Primo. [1986] 1989. Los hundidos y los salvados. Barcelona: Muchnik Editores, S. A.

Levi, Primo. [1947] 1999. Si esto es un hombre. Barcelona: Muchnik Editores, S. A.

Lustig, Oliver. [1982] 2002. Dicționar de lagăr. București: Editura Hasefer. 
Nomberg-Przytyk, Sara. [1966] 1985. True Tales from a Grotesque Land. North Carolina: The University of North Carolina Press.

Steinberg, Paul. 2004. Crónicas del mundo oscuro. Barcelona: Círculo de Lectores.

Venezia, Schlomo. [2007] 2010. Sonderkommando: el testimonio de un judio obligado a trabajar en las cámaras de gas. Barcelona: RBA Libros, S. A. 